\title{
Pubertal timing and breast cancer risk in the Sister Study cohort
}

\author{
Mandy Goldberg ${ }^{1}$, Aimee A. D'Aloisio², Katie M. O'Brien', Shanshan Zhao ${ }^{3}$ and Dale P. Sandler ${ }^{1 *}$ (D
}

\begin{abstract}
Background: Earlier age at menarche is an established risk factor for breast cancer. While age at menarche has been fairly stable over the past half-century, age at breast development (thelarche) has continued to decrease. Recently, earlier age at thelarche and a longer time between thelarche and menarche (pubertal tempo) were shown to be associated with increased breast cancer risk. Our objective was to examine how breast cancer risk was associated with pubertal timing and tempo in a prospective US cohort.

Methods: Women ages 35-74 years without a history of breast cancer, but who had a sister previously diagnosed with breast cancer, were enrolled in the Sister Study from 2003 to $2009(N=50,884)$. At enrollment, participants reported their ages at thelarche and menarche. Pubertal tempo was age at menarche minus age at thelarche. We estimated adjusted hazard ratios (HRs) and 95\% confidence intervals (Cls) for each pubertal milestone and risk of breast cancer (invasive or ductal carcinoma in situ) using Cox proportional hazards regression. We examined whether associations between age at thelarche and breast cancer risk were modified by birth cohort, race/ethnicity, weight at age 10, and extent of breast cancer family history, as characterized by a Bayesian score based on firstdegree family structure.
\end{abstract}

Results: During follow-up (mean = 9.3 years), 3295 eligible women were diagnosed with breast cancer. Early ages at thelarche ( $\mathrm{HR}=1.23,95 \% \mathrm{Cl} 1.03-1.46$ for $<10$ vs. $12-13$ years) and menarche ( $\mathrm{HR}=1.10,95 \% \mathrm{Cl} 1.01-1.20$ for $<12$ vs. 12-13 years) were positively associated with breast cancer risk. Pubertal tempo was not associated with breast cancer risk ( $H R=0.99,95 \% \mathrm{Cl} 0.97-1.02$ per 1-year longer tempo). When considering early thelarche $(<10$ years) and early menarche (<12 years) jointly, women with both had a 30\% greater risk of breast cancer compared with women with neither risk factor $(95 \% \mathrm{Cl} 1.07-1.57)$. The association between age at thelarche and breast cancer risk did not significantly vary by birth cohort, race/ethnicity, childhood weight, or Bayesian family history score.

Conclusions: Earlier ages at thelarche and menarche may enhance susceptibility to breast carcinogenesis. Age at thelarche is an important risk factor to consider given secular trends towards earlier development.

Keywords: Breast cancer, Puberty, Menarche, Breast development, Thelarche

\footnotetext{
*Correspondence: sandler@niehs.nih.gov

${ }^{1}$ Epidemiology Branch, National Institute of Environmental Health Sciences,

111 TW Alexander Dr, Research Triangle Park, NC 27709, USA

Full list of author information is available at the end of the article
}

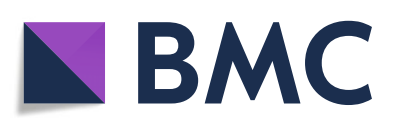

(c) This is a U.S. government work and not under copyright protection in the U.S.; foreign copyright protection may apply. 2020 Open Access This article is licensed under a Creative Commons Attribution 4.0 International License, which permits use, sharing, adaptation, distribution and reproduction in any medium or format, as long as you give appropriate credit to the original author(s) and the source, provide a link to the Creative Commons licence, and indicate if changes were made. The images or other third party material in this article are included in the article's Creative Commons licence, unless indicated otherwise in a credit line to the material. If material is not included in the article's Creative Commons licence and your intended use is not permitted by statutory regulation or exceeds the permitted use, you will need to obtain permission directly from the copyright holder. To view a copy of this licence, visit http://creativecommons.org/licenses/by/4.0/. The Creative Commons Public Domain Dedication waiver (http://creativecommons.org/publicdomain/zero/1.0/) applies to the data made available in this article, unless otherwise stated in a credit line to the data. 


\section{Background}

Earlier age at menarche is an established risk factor for breast cancer [1]. Age at menarche has historically decreased over time, but has remained fairly stable over the past halfcentury [2]. In contrast, age at onset of breast development (thelarche), which occurs on average 2-3 years before menarche [3], has continued to decline rapidly [2, 4-6]. Causes of this rapid decline in age at thelarche are not known, but increasing rates of childhood obesity and changes in environmental factors, such as exposure to endocrine-disrupting chemicals and psychosocial stressors, are hypothesized to contribute to this trend $[2,6,7]$. Puberty is a period of rapid breast development and a window of susceptibility for breast cancer risk, as the developing tissue may be particularly vulnerable to carcinogenesis [8]. Markers of pubertal development, such as thelarche and menarche, are associated with hormonal changes and breast growth [9] and are used to estimate the duration of this vulnerable period.

In contrast to age at menarche, few studies have examined other pubertal markers in relation to breast cancer risk, particularly age at thelarche. The timing of thelarche may be more biologically relevant to breast cancer risk than the timing of menarche as thelarche represents the onset of the vulnerable window of rapid breast development. Earlier thelarche and longer time period between thelarche and menarche were independently associated with a $20-30 \%$ increased risk of breast cancer in the Generations Study cohort [10]. In that study, earlier age at reaching adult height was also associated with increased breast cancer risk [10]. Age reached adult height is correlated with age at peak height velocity [11], a pubertal marker which occurs on average 1 year before menarche [12]. An inverse relationship between age reached adult height and breast cancer risk has also been observed in other [13-17], but not all [18], studies.

Our objective was to examine associations between pubertal markers and breast cancer risk in a prospective US cohort. These markers include age at thelarche, age at menarche, age at attainment of adult height, and the timing between these events. Given recent secular declines in age at thelarche $[2,4,5]$, we further evaluated whether factors associated with early thelarche, including race/ethnicity [4, 19], childhood weight [20], and extent of breast cancer family history [21], modified associations between age at thelarche and breast cancer risk.

\section{Methods}

\section{Study population}

The Sister Study is a prospective cohort designed to investigate environmental and genetic risk factors for breast cancer (for more details, see [22]). From 2003 to 2009, 50,884 women enrolled in the cohort. Women were eligible if they lived in the USA including Puerto Rico, were between the ages of 35 and 74 years, and did not have a personal history of breast cancer, but had a sister previously diagnosed with breast cancer.

Women completed a computer-assisted telephone interview at baseline which included information on demographics, medical and family history, and reproductive and lifestyle factors. During follow-up, women are asked to complete annual health updates and more extensive questionnaires every 2-3 years. Response rates have ranged from 91 to $96 \%$ throughout follow-up [22]. This analysis utilized Sister Study Data Release 7.2, including follow-up data up to September 15, 2017.

All participants provided written informed consent. The institutional review boards of the National Institute of Environmental Health Sciences, National Institutes of Health, and the Copernicus Group approved the study.

\section{Incident breast cancer}

Participants reported diagnoses of breast cancer on annual health updates and detailed follow-up questionnaires. Women who reported an incident breast cancer were asked for diagnostic information and permission to obtain medical records, including pathology reports. Medical records and/or pathology reports were obtained for more than $80 \%$ of women with a self-reported breast cancer. Agreement between self-reports and medical records has been high (positive predictive value >99\%) [23], so self-reports were used when medical records or pathology reports were not available. We examined incident breast cancer (invasive breast cancer or ductal carcinoma in situ (DCIS)) as the primary outcome of interest.

\section{Pubertal timing assessment}

During the baseline interview, women reported the age in years when they first noticed their breasts developing. Women who did not know the age were asked to report their grade in school, which we converted to age $(1.2 \%$ of the cohort reported grade). Women also reported the age in years and months when they had their first menstrual period. If age was unknown, women were asked to report their school grade $(0.3 \%$, converted to age) or if their period started before, around the same time as, or after other girls $(0.2 \%$, imputed as $11,12.5$, and 14 years $)$. We truncated age at menarche at years since few women reported months $(\sim 3 \%)$. Women responded to the question "How old were you when you first reached your full adult height? This is usually before the age of 20" with age in years. Women who did not know the age were given categorical options of 10-13, 14-17, and 18-20 years $(3.2 \%$, imputed as 12,16 , and 19 years). We calculated thelarche-menarche tempo as age at menarche minus age at thelarche and menarche-adult height tempo as age reached adult height minus age at menarche. 


\section{Analytic sample}

Of the 50,884 women enrolled in the cohort, we excluded 3 women who withdrew their data from the study; 75 women diagnosed with breast cancer prior to, at the same age as, or unknown relative to the completion of all baseline study components; and 3 women with uncertain cancer diagnoses (Additional File 1: Fig. S1). We also excluded women with unknown or missing ages at pubertal milestones $(n=738)$; women with implausibly late ages $(n=260)$, defined as $\geq 21$ for thelarche, $\geq 22$ for menarche, and $\geq 26$ for age reached adult height; and women who reported never having a menstrual period $(n=7)$. We selected these cut-offs to define implausible ages after exploring the distributions of self-reported ages at pubertal events in our data, as well as reviewing previous literature $[15,24]$ and considering the expected biological sequence of pubertal events. We excluded women with missing data on race/ethnicity or relative childhood family income $(n=112)$ for a final analytic sample of 49,686 women.

\section{Statistical analysis}

We examined the distributions of early-life characteristics and pubertal variables by age at thelarche. We used Spearman correlation coefficients to examine the relationships between continuous pubertal timing and tempo variables.

We used Cox proportional hazards regression with age as the time scale to estimate hazard ratios (HRs) and 95\% confidence intervals (CIs) for associations between pubertal exposures and breast cancer risk. Women accrued person-time from age at study enrollment until age at diagnosis of breast cancer or age at last study follow-up, loss to follow-up, or death. We tested for violations of the proportional hazard assumption using Wald or joint Wald tests of interaction terms between exposures and follow-up time. The assumption was not violated for the exposures of interest except where noted in table footnotes for secondary analyses.

We examined pubertal timing and tempo variables continuously and in categories. We considered models using the same categories examined in the Generations Study [10]. We stratified models by birth cohort in approximately 10 -year intervals to control for potential cohort effects. We then adjusted for race/ethnicity (nonHispanic white, non-Hispanic black, Hispanic, and others) and family income level growing up (well-off, middle income, low income, and poor), as reported by the participant at enrollment.

We modeled each pubertal exposure separately since exposures were correlated (Additional File 2: Table S1). We also present a mutually adjusted model including ages at thelarche and menarche and a model for age reached adult height adjusted for age at menarche. We present thelarche-menarche tempo models adjusted for age at thelarche and menarche-height tempo models adjusted for age at menarche to consider confounding by age at the first milestone. To further explore whether ages at thelarche and menarche were independent risk factors for breast cancer, we considered early thelarche $(<10$ years) and early menarche $(<12$ years) jointly using a four-category variable. We tested for interaction between early thelarche and early menarche through the Wald test of the cross-product term in a model that also included both main effects.

We examined whether associations between age at thelarche and breast cancer risk were modified by birth cohort, race/ethnicity, weight relative to peers at age 10 years (heavier vs. same weight or lighter), and height relative to peers at age 10 years (taller vs. same height or shorter) through stratification and tested for statistical heterogeneity using the Wald or joint Wald test. We excluded women with missing data for the modifier of interest in stratified models and also in models including main effects and interaction terms that tested for statistical heterogeneity ( $n=64$ women with missing childhood height and $n=124$ missing childhood weight). We also examined effect modification by familial risk using a continuous Bayesian family history score (BFHS) incorporating the family size, number of breast cancer cases in first-degree relatives, age at diagnosis for cases, and current age or age at death for non-cases (for more details, see [25, 26]). This score was developed in the Sister Study cohort. It ranges from 0 to 1 and can be interpreted as the family-specific pure lifetime breast cancer risk [25]. We stratified by BFHS, dichotomized at the median. We excluded women who reported at enrollment that they were adopted from the BFHS analyses $(n=183)$.

We considered heterogeneity in the association between age at thelarche and breast cancer risk by tumor invasiveness, estrogen receptor (ER) status, and menopausal status at diagnosis. For models examining invasive and DCIS disease separately, women with the alternate type were censored at age at diagnosis. For ER status, we included invasive and DCIS cases and censored women with the alternative subtype, with missing subtype information, or with a borderline test result at age at diagnosis. We calculated effect estimates and tested for statistical heterogeneity by invasiveness and ER status using fully adjusted joint Cox models additionally stratified by type [27]. We censored women who transitioned from pre-menopausal to post-menopausal during follow-up at age at menopause in models of premenopausal cancer. In models of post-menopausal cancer, women accrued person-time from age at cohort enrollment or age at menopause, whichever occurred later. We tested for heterogeneity by menopausal status using the Wald or joint Wald test.

We conducted sensitivity analyses to consider how error in the recall of age at thelarche may have biased 
associations between age at thelarche and breast cancer risk. Because thelarche is expected to occur prior to menarche, we assumed that thelarche occurred either 1 year or 2 years earlier than what they had reported for women who reported that thelarche occurred at the same age or later than menarche (tempo $\leq 0$ years) in order to quantify the bias arising from misclassification of age at thelarche. We also ran analyses restricting the study population to women more likely to have accurate recall. We limited models to women younger than 60 years at enrollment, as older women may be less likely to accurately report age at thelarche. In separate models, we excluded women with implausible thelarche-menarche tempo, as extreme tempos may reflect inaccurate reporting of age at thelarche. We defined implausible thelarchemenarche tempo in three ways: (1) tempo below the observed 5th percentile (menarche reported $>2$ years before thelarche) or above the 95th percentile (menarche reported $>3$ years after thelarche), (2) tempo $<0$ years (menarche reported before thelarche) or $>4$ years, and (3) tempo $\leq 0$ years (menarche reported at the same age or prior to thelarche).

We recalculated thelarche-menarche tempo after adjusting age at thelarche as described above in sensitivity analyses examining thelarche-menarche tempo and breast cancer risk. We also ran tempo analyses excluding women ages 60 and older at enrollment. In addition, we conducted a probabilistic bias analysis of the thelarchemenarche tempo and breast cancer relationship. To do so, we first evaluated the distribution of pubertal tempo among women who reported that menarche occurred at least 1 year after thelarche. We then assigned women with a thelarche-menarche tempo $\leq 0$ years a new tempo value based on a random draw from that distribution. We ran this simulation 20 times and combined the measures of association from fully adjusted Cox models across datasets using Rubin's rules [28].

We used robust variance estimates to account for correlations between sisters in the study sample. We conducted analyses using SAS 9.4 (SAS Institute Inc.).

\section{Results}

The mean age at thelarche in the sample was 12.2 years (median 12, range 4-20 years). Women with thelarche prior to 10 years of age were younger at enrollment and more likely to be non-Hispanic black or Hispanic, be heavier and taller than their peers in childhood, and grow up in a poor family (Table 1). Mean BFHS was similar across age at thelarche groups. Age at thelarche was positively correlated with age at menarche (Spearman $r=0.6)$ and age reached adult height $(r=0.3)$, and negatively correlated with time from thelarche to menarche $(r=-0.4)$ (Additional File 2: Table S1).

During a mean follow-up of 9.3 years, 3295 of 49,686 eligible women were diagnosed with invasive breast cancer or DCIS ( $78 \%$ and $22 \%$ of cases, respectively). Thelarche before 10 years of age was associated with a $23 \%$ greater risk of breast cancer compared with thelarche at $12-13$ years (95\% CI 1.03-1.46; Table 2). Each 1 -year delay in age at thelarche was associated with a $3 \%$ decrease in breast cancer risk (HR $=0.97,95 \%$ CI $0.95-$ 0.99). Earlier menarche was also associated with an increased risk of breast cancer $(\mathrm{HR}=1.10,95 \%$ CI 1.01-1.20 for $<12$ years compared with $12-13$ years; $\mathrm{HR}=0.96$, $95 \%$ CI $0.94-0.98$ for 1-year delay in age at menarche). These associations, particularly age at thelarche, were slightly attenuated when ages at thelarche and menarche were included in the same model.

Time from thelarche to menarche was not associated with breast cancer risk in models unadjusted for age at thelarche $(\mathrm{HR}=0.99,95 \% \mathrm{CI} \quad 0.97-1.02$ per 1-year increase in time from thelarche to menarche), but we observed an inverse association between tempo and risk after adjusting for age at thelarche ( $\mathrm{HR}=0.97,95 \%$ CI 0.94 1.00; Table 2). The HRs from the categorical model of tempo, although not statistically significant, also suggested an inverse trend after adjustment for age at thelarche. When considering early thelarche $(<10$ years $)$ and early menarche $(<12$ years) jointly, women with both had a $30 \%$ greater risk of breast cancer compared with women with neither risk factor (95\% CI 1.07-1.57; Fig. 1).

Reaching adult height at age 18 years or later was associated with a $13 \%$ decreased risk of breast cancer compared with reaching adult height at ages $15-17$ years ( $\mathrm{HR}=$ $0.87,95 \%$ CI $0.80-0.95$ ), but there was no increase in risk associated with reaching adult height at an early age (Table 2). These patterns were similar after adjustment for age at menarche, and there was no association between the time from menarche to adult height and breast cancer risk.

Among women at greater familial risk of breast cancer (BFHS $\geq$ median), early thelarche ( $<10$ years) was associated with increased breast cancer risk, while there was no significant decrease in risk associated with late thelarche (Fig. 2). In contrast, age at thelarche had an inverse, linear relationship with breast cancer risk in women with a BFHS below the median, and thelarche after 13 years was associated with a decreased risk. The heterogeneity $p$ values for the differences in the association between age at thelarche and breast cancer risk by median BFHS were 0.19 for the categorical exposure model and 0.06 for the continuous model. When BFHS was considered as a continuous variable, the heterogeneity $p$ values were 0.33 for the categorical age at thelarche model and 0.86 for the continuous model. The association between age at thelarche and breast cancer risk did not vary by birth cohort, race/ethnicity, or relative weight or height during childhood (Additional File 3: Table S2).

Associations between age at thelarche and breast cancer risk did not differ significantly by tumor invasiveness, 
Table 1 Participant characteristics by age at thelarche among 49,686 women enrolled in the Sister Study cohort

\begin{tabular}{|c|c|c|c|c|c|c|c|c|}
\hline & \multicolumn{8}{|c|}{ Age at thelarche } \\
\hline & \multicolumn{2}{|c|}{$<10(N=1743)$} & \multicolumn{2}{|c|}{$10-11(N=13,569)$} & \multicolumn{2}{|c|}{$12-13(N=25,762)$} & \multicolumn{2}{|c|}{$>13(N=8612)$} \\
\hline & Mean & SD & Mean & SD & Mean & SD & Mean & SD \\
\hline Age at baseline, years & 53.9 & 9.1 & 55.3 & 8.9 & 56.0 & 8.9 & 55.3 & 9.1 \\
\hline Bayesian family history score & 0.32 & 0.09 & 0.33 & 0.09 & 0.33 & 0.09 & 0.32 & 0.09 \\
\hline Age at menarche, years & 10.6 & 1.5 & 11.6 & 1.2 & 12.8 & 1.1 & 14.1 & 1.5 \\
\hline Time from thelarche to menarche, years & 1.8 & 1.6 & 1.0 & 1.2 & 0.4 & 1.1 & -0.6 & 1.6 \\
\hline Age reached adult height, years & 15.0 & 2.9 & 15.5 & 2.5 & 16.3 & 2.2 & 17.2 & 2.1 \\
\hline \multirow[t]{2}{*}{ Time from menarche to adult height, years } & 4.4 & 2.9 & 3.9 & 2.5 & 3.5 & 2.3 & 3.1 & 2.3 \\
\hline & $n$ & $\%$ & $n$ & $\%$ & $n$ & $\%$ & $n$ & $\%$ \\
\hline \multicolumn{9}{|l|}{ Birth cohort } \\
\hline 1928-1939 & 138 & 8 & 1432 & 11 & 3251 & 13 & 1047 & 12 \\
\hline 1940-1949 & 521 & 30 & 4472 & 33 & 8592 & 33 & 2602 & 30 \\
\hline 1950-1959 & 631 & 36 & 5052 & 37 & 9606 & 37 & 3243 & 38 \\
\hline 1960-1974 & 453 & 26 & 2613 & 19 & 4313 & 17 & 1720 & 20 \\
\hline \multicolumn{9}{|l|}{ Race/ethnicity } \\
\hline Non-Hispanic white & 1257 & 72 & 11,295 & 83 & 22,049 & 86 & 7050 & 82 \\
\hline Non-Hispanic black & 283 & 16 & 1198 & 9 & 1952 & 8 & 880 & 10 \\
\hline Hispanic & 154 & 9 & 735 & 5 & 1131 & 4 & 413 & 5 \\
\hline Others & 49 & 3 & 341 & 3 & 630 & 2 & 269 & 3 \\
\hline \multicolumn{9}{|l|}{ Family income level growing up } \\
\hline Well-off & 103 & 6 & 935 & 7 & 1628 & 6 & 511 & 6 \\
\hline Middle income & 952 & 55 & 8234 & 61 & 15,548 & 60 & 4940 & 57 \\
\hline Low income & 498 & 29 & 3388 & 25 & 6639 & 26 & 2362 & 27 \\
\hline Poor & 190 & 11 & 1012 & 7 & 1947 & 8 & 799 & 9 \\
\hline \multicolumn{9}{|l|}{ Relative weight to peers at age 10} \\
\hline Lighter & 298 & 17 & 3234 & 24 & 9197 & 36 & 4661 & 54 \\
\hline Same & 700 & 40 & 6616 & 49 & 12,595 & 49 & 3255 & 38 \\
\hline Heavier & 744 & 43 & 3693 & 27 & 3895 & 15 & 674 & 8 \\
\hline Missing & 1 & & 26 & & 75 & & 22 & \\
\hline \multicolumn{9}{|l|}{ Relative height to peers at age 10} \\
\hline Shorter & 332 & 19 & 2880 & 21 & 6612 & 26 & 2867 & 33 \\
\hline Same & 649 & 37 & 6071 & 45 & 12,424 & 48 & 3633 & 42 \\
\hline Taller & 760 & 44 & 4600 & 34 & 6687 & 26 & 2107 & 25 \\
\hline Missing & 2 & & 18 & & 39 & & 5 & \\
\hline
\end{tabular}

Column percentages are displayed. Missing data are excluded from percentages. Percentages may not add up to 100 due to rounding

menopausal status at diagnosis, or ER status (Additional File 4: Table S3). However, the association between early thelarche and breast cancer risk was only apparent for invasive (HR 1.32, 95\% CI 1.08-1.60) and post-menopausal $(\mathrm{HR}=1.32$, 95\% CI 1.09-1.60) disease.

The association between early thelarche and increased breast cancer risk was robust across sensitivity analyses with HRs ranging from 1.18 to 1.31 for < 10 years vs. $12-13$ years across models (Additional File 5: Table S4). The results for thelarche-menarche tempo and breast cancer risk were unchanged in sensitivity analyses using a recalculated tempo variable based on adjusting age at thelarche or excluding women ages $\geq 60$ years, or in probabilistic bias analyses, with HRs of 0.99-1.00 per 1-year longer tempo.

The inference across all pubertal exposures was similar when we used the same categories for pubertal timing and tempo as in the Generations Study (Additional File 6: Table S5) [10]. Although we did not observe an increased risk 
Table 2 Hazard ratios (HRs) and 95\% confidence intervals (Cls) for the associations between pubertal timing and tempo and incident breast cancer in the Sister Study cohort

\begin{tabular}{|c|c|c|c|c|c|c|c|c|}
\hline & \multirow{2}{*}{$\begin{array}{l}\text { Person- } \\
\text { years }\end{array}$} & \multirow{2}{*}{$\begin{array}{l}N \\
\text { cases }\end{array}$} & \multicolumn{2}{|c|}{ Minimally adjusted ${ }^{a}$} & \multicolumn{2}{|c|}{ Fully adjusted $^{b}$} & \multicolumn{2}{|c|}{ Mutually adjusted $^{c}$} \\
\hline & & & $\mathrm{HR}$ & $95 \% \mathrm{Cl}$ & $\mathrm{HR}$ & $95 \% \mathrm{Cl}$ & $\mathrm{HR}$ & $95 \% \mathrm{Cl}$ \\
\hline \multicolumn{9}{|l|}{ Age at pubertal milestone } \\
\hline \multicolumn{9}{|l|}{ Age at thelarche } \\
\hline$<10$ years & 15,806 & 135 & 1.22 & $1.03,1.46$ & 1.23 & $1.03,1.46$ & 1.15 & $0.95,1.39$ \\
\hline 10-11 years & 125,803 & 921 & 1.02 & $0.95,1.11$ & 1.03 & $0.95,1.11$ & 0.98 & $0.90,1.08$ \\
\hline $12-13$ years & 238,712 & 1725 & 1.00 & Referent & 1.00 & Referent & 1.00 & Referent \\
\hline$>13$ years & 79,405 & 514 & 0.91 & $0.82,1.00$ & 0.91 & $0.82,1.00$ & 0.93 & $0.84,1.04$ \\
\hline Continuous (per 1-year later) & 459,726 & 3295 & 0.97 & $0.95,0.99$ & 0.97 & $0.95,0.99$ & 0.99 & $0.96,1.02$ \\
\hline \multicolumn{9}{|l|}{ Age at menarche } \\
\hline$<12$ years & 93,485 & 742 & 1.10 & $1.01,1.20$ & 1.10 & $1.01,1.20$ & 1.09 & $0.99,1.20$ \\
\hline $12-13$ years & 258,756 & 1849 & 1.00 & Referent & 1.00 & Referent & 1.00 & Referent \\
\hline$>13$ years & 107,484 & 704 & 0.92 & $0.84,1.00$ & 0.92 & $0.85,1.01$ & 0.95 & $0.86,1.04$ \\
\hline Continuous (per 1-year later) & 459,726 & 3295 & 0.96 & $0.94,0.98$ & 0.96 & $0.94,0.98$ & 0.97 & $0.94,1.00$ \\
\hline \multicolumn{9}{|l|}{ Age reached adult height } \\
\hline$<15$ years & 116,762 & 878 & 0.97 & $0.89,1.06$ & 0.97 & $0.89,1.06$ & 0.94 & $0.86,1.03$ \\
\hline $15-17$ years & 188,674 & 1412 & 1.00 & Referent & 1.00 & Referent & 1.00 & Referent \\
\hline$>17$ years & 154,289 & 1005 & 0.87 & $0.80,0.94$ & 0.87 & $0.80,0.95$ & 0.88 & $0.81,0.95$ \\
\hline Continuous (per 1-year later) & 459,726 & 3295 & 0.99 & $0.97,1.00$ & 0.99 & $0.97,1.00$ & 0.99 & $0.98,1.01$ \\
\hline \multicolumn{9}{|l|}{ Pubertal tempo } \\
\hline \multicolumn{9}{|l|}{ Time from thelarche to menarche } \\
\hline$<0$ years & 81,423 & 592 & 1.01 & $0.92,1.11$ & 1.01 & $0.92,1.11$ & 1.06 & $0.95,1.17$ \\
\hline 0 years & 169,355 & 1239 & 1.00 & Referent & 1.00 & Referent & 1.00 & Referent \\
\hline 1 year & 130,298 & 913 & 0.97 & $0.89,1.05$ & 0.96 & $0.89,1.05$ & 0.95 & $0.87,1.03$ \\
\hline$>1$ year & 78,650 & 551 & 0.99 & $0.90,1.10$ & 1.00 & $0.90,1.10$ & 0.96 & $0.86,1.06$ \\
\hline Continuous (per 1-year longer) & 459,726 & 3295 & 0.99 & $0.97,1.02$ & 0.99 & $0.97,1.02$ & 0.97 & $0.94,1.00$ \\
\hline \multicolumn{9}{|c|}{ Time from menarche to adult height } \\
\hline$<2$ years & 95,450 & 702 & 0.98 & $0.89,1.08$ & 0.98 & $0.89,1.08$ & 0.99 & $0.90,1.09$ \\
\hline $2-3$ years & 137,145 & 973 & 0.97 & $0.89,1.06$ & 0.97 & $0.89,1.06$ & 0.98 & $0.89,1.07$ \\
\hline $4-5$ years & 134,086 & 971 & 1.00 & Referent & 1.00 & Referent & 1.00 & Referent \\
\hline$>5$ years & 93,045 & 649 & 0.96 & $0.87,1.06$ & 0.96 & $0.87,1.06$ & 0.93 & $0.84,1.03$ \\
\hline Continuous (per 1-year longer) & 459,726 & 3295 & 1.00 & $0.99,1.02$ & 1.00 & $0.99,1.02$ & 0.99 & $0.98,1.01$ \\
\hline
\end{tabular}

$N=49,686$ women included in each model. No violations of proportional hazards assumption for any of the exposures of interest

${ }^{a}$ Adjusted for attained age as the underlying time scale and stratified by birth cohort

${ }^{\mathrm{b}}$ Additionally adjusted for race/ethnicity and family income level growing up

cFully adjusted model with mutual adjustment for ages at thelarche and menarche. Age reached adult height is adjusted for age at menarche. Pubertal tempo models are adjusted for age at thelarche (for the thelarche-menarche model) and age at menarche (for the menarche-height model)

associated with early thelarche when defined as $\leq 10$ years compared with $11-12$ years ( $\mathrm{HR}=1.02$, 95\% CI 0.92-1.13), thelarche at $\geq 13$ years was associated with decreased breast cancer risk (HR 0.93, 95\% CI 0.86-1.00) and breast cancer risk decreased with each category increase in age at thelarche.

\section{Discussion}

Earlier ages at thelarche and menarche were associated with increased breast cancer risk in a prospective cohort of US women with a family history of breast cancer. Having both early thelarche and early menarche was associated with an even greater increase in risk than experiencing only one of these pubertal events at an early age. Reaching adult height at a later age was associated with a modest decrease in breast cancer risk. Neither time from thelarche to menarche nor time from menarche to attainment of adult height was associated with breast cancer risk.

Although breast development begins in utero, the majority occurs after birth and is initiated during puberty 


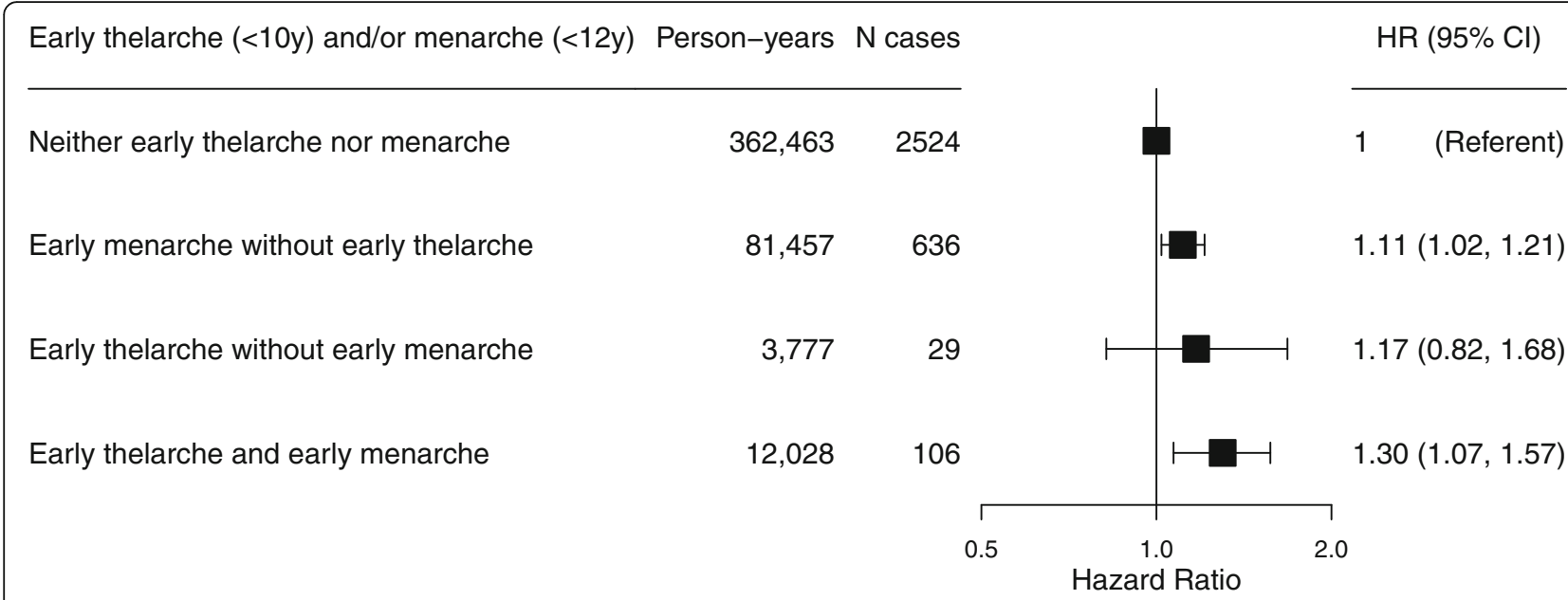

Fig. 1 Associations between early thelarche, early menarche, and incident breast cancer in the Sister Study cohort. $N=49,686$ women. Early thelarche is defined as $<10$ years (vs. $\geq 10$ years), and early menarche is defined as $<12$ years (vs. $\geq 12$ years). Estimates are adjusted for attained age, race/ ethnicity, and family income level growing up and stratified by birth cohort. $p$ for interaction between early thelarche and early menarche $=0.99$. Hazard ratios (HRs) and 95\% confidence intervals (Cls) are plotted on the log scale

[29]. Puberty is triggered by the re-activation of the hypothalamic-pituitary-gonadal axis in childhood. This causes an increase in endogenous hormones; the appearance of physical markers of pubertal development, including breast growth and the pubertal growth spurt in height; and the onset of menses in girls (reviewed in [30]). Hormones and growth factors, particularly estrogen and insulin-like growth factor-1 (IGF-1), work together to regulate breast development during puberty [31, 32]. Rapid breast cell proliferation occurs, including ductal

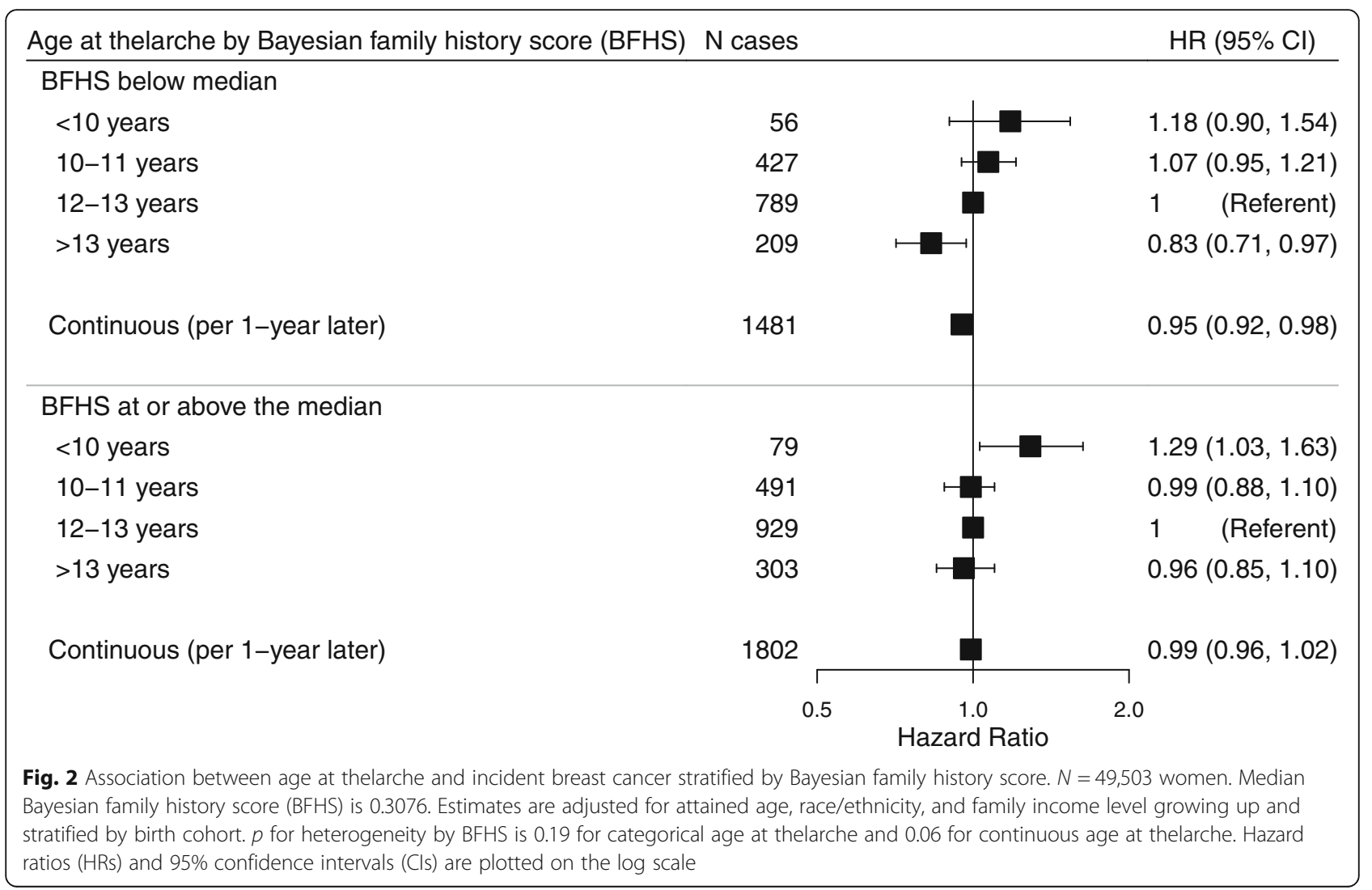


branching and the formation of terminal end buds [29, 32]. These later differentiate into terminal ductal lobular units, the structure within the breast where most cancers originate $[29,33]$. However, breast cells do not fully differentiate until the completion of a full-term pregnancy and lactation $[8,31]$.

Earlier puberty may increase breast cancer risk by increasing the time that breast cells are in a highly proliferative, undifferentiated state that leaves them more susceptible to carcinogenesis [34]. The time between thelarche and menarche is considered biologically relevant to breast cancer risk since the majority of ductal development occurs during this window [10, 29]. Girls who enter puberty earlier experience increases in endogenous hormones at earlier ages. Peak height growth is associated with increased levels of IGF-1, a mitogen that stimulates breast development [35]. Estrogen and progesterone levels rise after menarche, increasing breast cell proliferation $[29,36]$. Higher levels of estrogen [37, 38] and IGF-1 [39] in adulthood have been associated with increased breast cancer risk. Earlier and potentially prolonged exposure to high levels during puberty may increase breast cancer risk as well.

We found that earlier age at thelarche, which represents the onset of this period of rapid breast development, was associated with an increased risk of breast cancer. The $23 \%$ increased risk associated with thelarche prior to 10 years of age is identical to the association observed in the Generations Study (HR $=1.23,95 \%$ CI $1.02-1.48$ for $\leq 10$ years vs. 11-12 years) [10], albeit using different cut-offs to define early thelarche. Age at thelarche was inversely associated with the risk of both ER+ and ER- cancers. To our knowledge, the association between age at thelarche and breast cancer risk has not been previously examined by tumor receptor status. The lack of heterogeneity in the age at thelarche association by subtype is consistent with literature suggesting that age at menarche is inversely associated with both hormone receptor-positive and hormone receptornegative disease [40]. We did not observe significant differences by menopausal status or tumor invasiveness, consistent with the findings in the Generations Study [10]. The positive associations between early thelarche and breast cancer risk in our study were limited to postmenopausal breast cancer and invasive disease, though there were relatively few pre-menopausal or DCIS cases.

Age at thelarche has declined over time [2], and this secular trend is evident in our cohort as women born after 1960 were more likely to report earlier thelarche. The inverse association between age at thelarche and breast cancer risk was observed across the birth cohorts in our study, spanning from the 1930s to the 1970s. Data from the USA and Europe suggest that age at thelarche has continued to decline, with a mean age of less than 10 years observed in recent pubertal cohorts $[4,5]$. Given this continued secular decline, early age at thelarche may contribute to future trends in breast cancer incidence.

The attributable risk of breast cancer due to earlier puberty may be higher in populations where early thelarche is more common. Age at thelarche is earlier, on average, in black and Hispanic girls in the USA than in non-Hispanic white girls $[4,19]$. In our cohort, nonHispanic black and Hispanic women were more likely to report early thelarche than non-Hispanic white women. Thus, while the association between age at thelarche and breast cancer risk did not vary meaningfully by race/ethnicity, it may still contribute differentially to the burden of breast cancer among women of different racial/ethnic groups. Girls that are overweight in childhood are more likely to experience earlier thelarche [20], but childhood adiposity is associated with decreased breast cancer risk $[41,42]$. Our finding that the association between age at thelarche and breast cancer risk was not modified by relative childhood weight suggests that early puberty and childhood weight affect risk through different pathways and that early thelarche should be considered a risk factor in both overweight and non-overweight girls. The relationship between earlier thelarche and increased breast cancer risk was observed in women that were taller than their peers in childhood, a group at increased risk of both early puberty [43] and breast cancer [44], as well as in women that were the same height or shorter than their peers.

Women in our study have at least one sister with breast cancer and have, on average, approximately twice the risk of breast cancer than women without a first-degree family history [45]. The consistency of the age at thelarche association in our cohort and the Generations Study, in which only $15 \%$ of the study population had a first-degree family history [10], suggests that early thelarche is a risk factor for breast cancer in women with and without a family history. Studies of twin pairs have suggested that earlier thelarche may be associated with increased breast cancer risk and earlier age at onset among women with a family history of breast cancer $[46,47]$. In our study, early thelarche $(<10$ years) was associated with increased breast cancer risk across the range of first-degree family history captured through the BFHS, although the association was statistically significant and the point estimate higher for women with a BFHS at or above the median. For late age at thelarche ( $>13$ years), hazard ratios in both groups suggested an association with decreased breast cancer risk, but the estimate for women with a BFHS below the median was further from the null and statistically significant. While this apparent difference in results is intriguing, there is no evidence that the findings differ significantly by family history score, so we are reluctant to speculate about possible 
biological explanations. Furthermore, the mean BFHS did not vary by age at thelarche. However, since women with a family history have a greater underlying risk of breast cancer, the increased risk associated with early thelarche will be larger on an absolute scale in these women compared with women who are not at increased familial risk [48].

Consistent with prior studies [1], we also observed an inverse association between age at menarche and breast cancer risk. It is difficult to disentangle the influence of early thelarche from early menarche, given the high correlation between ages at these events. However, we observed a greater increased risk of breast cancer associated with experiencing both milestones at an early age than experiencing early thelarche or early menarche only, suggesting that the timing of both markers contributes to risk. While age at menarche is often collected in research settings, age at thelarche is not commonly assessed. More studies should collect data on age at thelarche to further explore the independent contribution of age at thelarche to breast cancer risk and examine the utility of using this information to improve risk assessment. In the future, routine collection of age at thelarche in clinical settings and inclusion in risk assessment models may help to identify high-risk women that may benefit from increased screening or risk reduction measures.

We did not observe an association between the time from thelarche to menarche, or tempo, and breast cancer risk, in contrast to the positive association observed in the Generations Study [10]. Our cohort was older on average than the Generations cohort at enrollment, but the inference was unchanged when we limited the tempo analysis to women less than 60 years at baseline. Misreporting of pubertal timing, particularly age at thelarche, may have limited our ability to detect an association between thelarche-menarche tempo and breast cancer risk. Age at menarche can be reliably recalled in adulthood [49], but the reliability and validity of recalled age at thelarche is not known. The mean age at menarche in the cohort was 12.6 years, which is similar to data from NHANES [50]. The mean age at thelarche was 12.2 years and the mean thelarche-menarche tempo was 0.4 years in our cohort. In comparison, longitudinal studies of women born in the same birth cohorts as our study population observed that thelarche occurred on average around 11 years of age and approximately 2 years prior to menarche (reviewed in [3]). This suggests that women tended to recall a later age at thelarche than when it occurred, leading to an underestimation of pubertal tempo and a potential bias towards the null in our analysis of tempo and breast cancer risk. In our cohort, $37 \%$ of women reported that thelarche occurred at the same age as menarche, which likely reflects misreporting of age at thelarche as well as reduced precision of the tempo assessment since thelarche and menarche were reported to the nearest year. Since women are regularly asked about age at menarche but not thelarche, it is possible that women who were reluctant to answer "do not know" assumed thelarche must have been at around the same time as menarche. In sensitivity analyses, we considered whether inaccuracies in reporting age at thelarche explained the lack of association between tempo and breast cancer risk but results were unchanged. However, tempo was positively associated with breast density in a subset of the Generations Study [51] and in the prospective Dietary Intervention Study in Children follow-up study [52]. We therefore would not rule out an association between tempo and breast cancer risk but suggest that additional studies with prospective assessments of thelarche and menarche will be necessary to limit measurement error in estimates of the association between tempo and breast cancer risk.

We also observed a decreased risk of breast cancer associated with reaching adult height at a later age. Our findings are consistent with previous studies [10, 13-17], though no association was observed in pre-menopausal women in the Nurses' Health Study II cohort [18]. We did not observe an association between time from menarche to adult height and breast cancer risk, similar to others $[10,18]$. Earlier age reaching adult height is correlated with an earlier age at peak height velocity and a faster rate of height growth [11], which have both been associated with increased breast cancer risk [44].

Strengths of this study include the large sample size; prospective design, which limited differential recall of pubertal timing by breast cancer status; and inclusion of multiple pubertal markers. We were able to assess whether the association between age at thelarche and breast cancer risk varied by breast cancer characteristics including ER status. We further examined effect modification by factors associated with early thelarche, including race/ethnicity, childhood body size, and extent of breast cancer family history, which to our knowledge have not been investigated previously.

Analyses were limited by recalled data on ages at pubertal milestones, which may be reported with error. We categorized the exposures to limit bias resulting from measurement error, although we also examined ages at pubertal milestones continuously to facilitate comparisons with prior studies. The validity of age at menarche, for example, has been shown to be improved when categorized as early, normal, and late [53]. Age at thelarche in particular may be difficult for women to accurately recall in adulthood. Sensitivity analyses to assess the influence of misclassification of age at thelarche suggest that inaccurate recall does not account for the association observed between earlier thelarche and increased breast cancer risk, as we observed minimal attenuation of the hazard ratios in quantitative bias analyses. However, inaccuracies in 
self-reports of age at thelarche may have limited our ability to detect an association between thelarche-menarche tempo and breast cancer risk. As a derived variable, tempo may be more sensitive to reporting errors in the exact ages of thelarche and menarche than analyses examining the timing of these milestones individually. In addition, multiple testing of correlated pubertal exposures increased the likelihood of a false-positive finding. We also had reduced precision in stratified analyses for small subgroups, such as some racial/ethnic groups. We did not have the temporal data necessary to examine whether early-life body size confounded or mediated the relationship between age at thelarche and breast cancer risk since body size was reported for age 10 and age at thelarche ranged from 4 to 20 years. Although all women in our cohort have a sister with breast cancer, the consistency of our findings with other studies suggests that our results may still be generalizable to women without a family history.

\section{Conclusions}

Our findings suggest that earlier age at attainment of pubertal milestones may enhance susceptibility to breast carcinogenesis. Early age at thelarche is an emerging breast cancer risk factor that may have considerable public health impact, given secular declines in age at thelarche in the USA and abroad. Studies with prospective measures of pubertal tempo are needed to clarify whether the time between thelarche and menarche is a particularly vulnerable period in relation to breast cancer risk.

\section{Supplementary information}

Supplementary information accompanies this paper at https://doi.org/10. 1186/s13058-020-01326-2.

Additional file 1: Figure S1. Flow chart of eligible study population. Additional file 2: Table S1. Spearman correlations between pubertal variables

Additional file 3: Table S2. Hazard ratios (HRs) and 95\% confidence intervals (Cls) for the association between age at thelarche and incident breast cancer in the Sister Study cohort stratified by characteristics associated with age at thelarche

Additional file 4: Table S3. Hazard ratios (HRs) and 95\% confidence intervals (Cls) for the association between age at thelarche and incident breast cancer in the Sister Study cohort by breast cancer characteristics

Additional file 5: Table S4. Sensitivity analyses for the association between age at thelarche and incident breast cancer in the Sister Study cohort

Additional file 6: Table S5. Hazard ratios (HRs) and 95\% confidence intervals (Cls) for the associations between pubertal timing and tempo and incident breast cancer in the Sister Study cohort using the same categories as in the Generations Study [10]

\section{Abbreviations}

BFHS: Bayesian family history score; CI: Confidence interval; DCIS: Ductal carcinoma in situ; ER: Estrogen receptor; HR: Hazard ratio; IGF-1: Insulin-like growth factor-1

\section{Acknowledgements}

The authors appreciate the helpful comments of Drs. Helen Chin and Nicole Niehoff.

\section{Authors' contributions}

MG conducted and interpreted the data analyses and drafted the initial manuscript. AAD and KMO participated in the data preparation, analysis, and interpretation. SZ created the Bayesian family history score and contributed to family history analyses and interpretation. DPS designed the parent study, acquired the data, and oversaw the statistical analyses, interpretation, and writing. All authors critically reviewed and approved the final manuscript.

\section{Funding}

This work was supported by the Intramural Research Program of the National Institutes of Health, National Institute of Environmental Health Sciences (Z01-ES044005 to DPS).

\section{Availability of data and materials}

Requests for data, including the data used in this manuscript, are welcome. De-identified data is made available upon request as described on the study website (https://sisterstudy.niehs.nih.gov/English/data-requests.htm). The data sharing policy was developed to protect the privacy of study participants and is consistent with study informed consent documents as approved by the NIEHS Institutional Review Board.

\section{Ethics approval and consent to participate}

All participants provided written informed consent. The institutional review boards of the National Institute of Environmental Health Sciences, National Institutes of Health, and the Copernicus Group approved the study.

\section{Consent for publication \\ Not applicable.}

\section{Competing interests}

The authors declare that they have no competing interests.

\section{Author details}

${ }^{1}$ Epidemiology Branch, National Institute of Environmental Health Sciences, 111 TW Alexander Dr, Research Triangle Park, NC 27709, USA. ${ }^{2}$ Social and Scientific Systems, Durham, NC, USA. ${ }^{3}$ Biostatistics \& Computational Biology Branch, National Institute of Environmental Health Sciences, Research Triangle Park, NC, USA.

Received: 22 April 2020 Accepted: 3 August 2020

Published online: 27 October 2020

\section{References}

1. Collaborative Group on Hormonal Factors in Breast Cancer. Menarche, menopause, and breast cancer risk: individual participant meta-analysis, including 118964 women with breast cancer from 117 epidemiological studies. Lancet Oncol. 2012;13(11):1141-51.

2. Lee $Y$, Styne D. Influences on the onset and tempo of puberty in human beings and implications for adolescent psychological development. Horm Behav. 2013;64(2):250-61.

3. Biro FM, Pajak A, Wolff MS, Pinney SM, Windham GC, Galvez MP, et al. Age of menarche in a longitudinal US cohort. J Pediatr Adolesc Gynecol. 2018; 31(4):339-45.

4. Biro FM, Greenspan LC, Galvez MP, Pinney SM, Teitelbaum S, Windham GC, et al. Onset of breast development in a longitudinal cohort. Pediatrics. 2013; 132(6):1019-27.

5. Aksglaede L, Sørensen K, Petersen JH, Skakkebæk NE, Juul A. Recent decline in age at breast development: the Copenhagen Puberty Study. Pediatrics. 2009;123(5):e932-9.

6. Eckert-Lind C, Busch AS, Petersen JH, Biro FM, Butler G, Bräuner EV, et al. Worldwide secular trends in age at pubertal onset assessed by breast development among girls: a systematic review and meta-analysis. JAMA Pediatr. 2020;174(4):e195881.

7. Parent AS, Teilmann G, Juul A, Skakkebaek NE, Toppari J, Bourguignon JP. The timing of normal puberty and the age limits of sexual precocity: variations around the world, secular trends, and changes after migration. Endocr Rev. 2003;24(5):668-93. 
8. Martinson HA, Lyons TR, Giles ED, Borges VF, Schedin P. Developmental windows of breast cancer risk provide opportunities for targeted chemoprevention. Exp Cell Res. 2013;319:1671-8.

9. Biro FM, Deardorff J. Identifying opportunities for cancer prevention during preadolescence and adolescence: puberty as a window of susceptibility. J Adolesc Health. 2013;52(5 Suppl):S15-20.

10. Bodicoat DH, Schoemaker MJ, Jones ME, McFadden E, Griffin J, Ashworth A, et al. Timing of pubertal stages and breast cancer risk: the Breakthrough Generations Study. Breast Cancer Res. 2014;16(1):R18.

11. Tanner JM, Davies PS. Clinical longitudinal standards for height and height velocity for North American children. J Pediatr. 1985;107(3):317-29.

12. Karlberg J. Secular trends in pubertal development. Horm Res. 2002; 57(Suppl 2):19-30.

13. Bertrand KA, Gerlovin H, Bethea TN, Palmer JR. Pubertal growth and adult height in relation to breast cancer risk in African American women. Int J Cancer. 2017;141(12):2462-70.

14. Li Cl, Malone KE, White E, Daling JR. Age when maximum height is reached as a risk factor for breast cancer among young U.S. women. Epidemiology. 1997:8(5):559-65.

15. Li Cl, Littman AJ, White E. Relationship between age maximum height is attained, age at menarche, and age at first full-term birth and breast cancer risk. Cancer Epidemiol Biomark Prev. 2007;16(10):2144-9.

16. Beaber EF, Holt VL, Malone KE, Porter PL, Daling JR, Li Cl. Reproductive factors, age at maximum height, and risk of three histologic types of breast cancer. Cancer Epidemiol Biomark Prev. 2008;17(12):3427-34.

17. $\mathrm{Li} \mathrm{Cl}$. Anthropometric variables in relation to risk of breast cancer in middleaged women. Int J Epidemiol. 2000;29(2):208-13.

18. Baer HJ, Rich-Edwards JW, Colditz GA, Hunter DJ, Willett WC, Michels KB. Adult height, age at attained height, and incidence of breast cancer in premenopausal women. Int J Cancer. 2006;119(9):2231-5.

19. Herman-Giddens ME, Slora EJ, Wasserman RC, Bourdony CJ, Bhapkar MV, Koch GG, et al. Secondary sexual characteristics and menses in young girls seen in office practice: a study from the pediatric research in office settings network. Pediatrics. 1997;99(4):505-12.

20. Kaplowitz PB. Link between body fat and the timing of puberty. Pediatrics. 2008;121(Suppl):S208-17.

21. Terry MB, Keegan THM, Houghton LC, Goldberg M, Andrulis IL, Daly MB, et al. Pubertal development in girls by breast cancer family history: the LEGACY girls cohort. Breast Cancer Res. 2017;19(1):69.

22. Sandler DP, Hodgson ME, Deming-Halverson SL, Juras PS, D'Aloisio AA, Suarez LM, et al. The Sister Study cohort: baseline methods and participant characteristics. Environ Health Perspect. 2017;125(12):127003.

23. D'Aloisio AA, Nichols HB, Hodgson ME, Deming-Halverson SL, Sandler DP. Validity of self-reported breast cancer characteristics in a nationwide cohort of women with a family history of breast cancer. BMC Cancer. 2017;17(1):692.

24. Rosner B, Colditz GA, Willett WC. Reproductive risk factors in a prospective study of breast cancer: the Nurses' Health Study. Am J Epidemiol. 1994; 139(8):819-35

25. Jiang $Y$, Weinberg CR, Sandler DP, Zhao S. Use of detailed family history data to improve risk prediction, with application to breast cancer screening. PLoS One. 2019;14(12):e0226407.

26. Niehoff NM, Nichols HB, Zhao S, White AJ, Sandler DP. Adult physical activity and breast cancer risk in women with a family history of breast cancer. Cancer Epidemiol Biomark Prev. 2019;28(1):51-8.

27. Xue X, Kim MY, Gaudet MM, Park Y, Heo M, Hollenbeck AR, et al. A comparison of the polytomous logistic regression and joint Cox proportional hazards models for evaluating multiple disease subtypes in prospective cohort studies. Cancer Epidemiol Biomark Prev. 2013; 22(2):275-85.

28. Rubin DB. Multiple imputation for nonresponse in surveys. New York: Wiley; 1987.

29. Russo J, Russo $1 H$. Development of the human breast. Maturitas. 2004; 49:2-15.

30. Abreu AP, Kaiser UB. Pubertal development and regulation. Lancet Diabetes Endocrinol. 2016;4(3):254-64.

31. Macias H, Hinck L. Mammary gland development. Wiley Interdiscip Rev Dev Biol. 2012;1(4):533-57.

32. Rudel RA, Fenton SE, Ackerman JM, Euling SY, Makris SL. Environmental exposures and mammary gland development: state of the science, public health implications, and research recommendations. Environ Health Perspect. 2011;119(8):1053-61.
33. Oh $\mathrm{H}$, Bodelon $\mathrm{C}$, Palakal M, Chatterjee $\mathrm{N}$, Sherman ME, Linville L, et al. Ages at menarche- and menopause-related genetic variants in relation to terminal duct lobular unit involution in normal breast tissue. Breast Cancer Res Treat. 2016;158(2):341-50.

34. Fenton SE. Endocrine-disrupting compounds and mammary gland development: early exposure and later life consequences. Endocrinology. 2006;147(6 Suppl):S18-24.

35. Sachdev D, Yee D. The IGF system and breast cancer. Endocr Relat Cancer. 2001;8(3):197-209.

36. Eden JA. Breast cancer, stem cells and sex hormones. Part 2: the impact of the reproductive years and pregnancy. Maturitas. 2010;67(3):215-8.

37. Endogenous Hormones and Breast Cancer Collaborative Group. Sex hormones and risk of breast cancer in premenopausal women: a collaborative reanalysis of individual participant data from seven prospective studies. Lancet Oncol. 2013;14(10):1009-19.

38. Endogenous Hormones and Breast Cancer Collaborative Group. Endogenous sex hormones and breast cancer in postmenopausal women: reanalysis of nine prospective studies. J Natl Cancer Inst. 2002; 94(8):606-16.

39. Endogenous Hormones and Breast Cancer Collaborative Group. Insulin-like growth factor 1 (IGF1), IGF binding protein 3 (IGFBP3), and breast cancer risk: pooled individual data analysis of 17 prospective studies. Lancet Oncol. 2010;11(6):530-42.

40. Gaudet MM, Gierach GL, Carter BD, Luo J, Milne RL, Weiderpass E, et al. Pooled analysis of nine cohorts reveals breast cancer risk factors by tumor molecular subtype. Cancer Res. 2018;78(20):6011-21.

41. Le Marchand L, Kolonel LN, Earle ME, Mi MP. Body size at different periods of life and breast cancer risk. Am J Epidemiol. 1988;128(1):137-52.

42. Hilakivi-Clarke L. Tallness and overweight during childhood have opposing effects on breast cancer risk. Br J Cancer. 2001;85(11):1680-4.

43. Davison KK, Susman EJ, Birch LL. Percent body fat at age 5 predicts earlier pubertal development among girls at age 9. Pediatrics. 2003;111(4 Pt 1): 815-21.

44. Ahlgren M, Melbye M, Wohlfahrt J, Sorensen TI. Growth patterns and the risk of breast cancer in women. N Engl J Med. 2004;351(16):1619-26.

45. Collaborative Group on Hormonal Factors in Breast Cancer. Familial breast cancer: collaborative reanalysis of individual data from 52 epidemiological studies including 58209 women with breast cancer and 101986 women without the disease. Lancet. 2001;358(9291):1389-99.

46. Swerdlow AJ, De Stavola BL, Floderus B, Holm NV, Kaprio J, Verkasalo PK, et al. Risk factors for breast cancer at young ages in twins: an international population-based study. J Natl Cancer Inst. 2002;94(16):1238-46.

47. Hamilton AS, Mack TM. Puberty and genetic susceptibility to breast cancer in a case-control study in twins. N Engl J Med. 2003;348(23):2313-22.

48. Hopper JL. Genetics for population and public health. Int J Epidemiol. 2017; 46(1):8-11.

49. Must A, Phillips SM, Naumova EN, Blum M, Harris S, Dawson-Hughes B, et al. Recall of early menstrual history and menarcheal body size: after 30 years, how well do women remember? Am J Epidemiol. 2002;155(7):672-9.

50. McDowell MA, Brody DJ, Hughes JP. Has age at menarche changed? Results from the National Health and Nutrition Examination Survey (NHANES) 19992004. J Adolesc Health. 2007;40(3):227-31.

51. Schoemaker MJ, Jones ME, Allen S, Hoare J, Ashworth A, Dowsett M, et al. Childhood body size and pubertal timing in relation to adult mammographic density phenotype. Breast Cancer Res. 2017;19(1):13.

52. Houghton LC, Jung S, Troisi R, Leblanc ES, Snetselaar LG, Hylton NM, et al. Pubertal timing and breast density in young women: a prospective cohort study. Breast Cancer Res. 2019;21(1):122.

53. Cooper R, Blell M, Hardy R, Black S, Pollard TM, Wadsworth MEJ, et al. Validity of age at menarche self-reported in adulthood. J Epidemiol Community Health. 2006;60(11):993-7.

\section{Publisher's Note}

Springer Nature remains neutral with regard to jurisdictional claims in published maps and institutional affiliations. 